\title{
Ingénierie génétique et spermatogenèse
}

\author{
D. ESCALIER
}

INSERM U25, Hôpital Necher, 149 rue de Sèvres, 75743 Paris Cedex 15 escalier@necker.fr

\section{RESUME}

L'ingéniérie génétique permet une analyse fonctionnelle des gènes impliqués dans la spermatogenèse. Dans de nombreux cas, l'atteinte de la spermatogenèse a été fortuite, confirmant la grande sensibilité de cette fonction vis à vis des altérations génétiques. Les études ont déjà portées sur près de cent de ces gènes et ont permis entre autres : l'étude des facteurs impliqués dans la méiose, communs ou non aux deux sexes, et leurs rapports éventuels avec le cancer et l'apoptose ; de faire un premier tri entre les facteurs qui peuvent être impliqués dans des stérilités et ceux qui peuvent être compensés par redondance génétique ; la dissection de mécanismes de la spermiogenèse ; l'analyse des processus impliqués dans la réponse au choc thermique et la barrière hémato-testiculaire. Les résultats s'étendent à d'autres niveaux de la biologie de la reproduction pour les deux sexes, et laissent apparaître des maladies pouvant être associées à une stérilité masculine. Les données montrent aussi l'importance des dissociations entre le génotype et le phénotype des spermatozoïdes et soulèvent la question de différences fonctionnelles interspécifiques de gènes homologues.

Mots-clés : souris transgénique, inactivation génique, méiose, spermatogenèse, spermatozoïdes, testicule
Actuellement environ 100 gènes exprimés au niveau testiculaire ont été étudiés par génie génétique, et pour environ 90 d'entre eux, une modification de leur expression a induit des perturbations de la spermatogenèse. Les phénotypes obtenus par induction d'anomalies de la spermatogenèse par génie génétique sont répertoriés dans plusieurs revues $[10,14]$. Ils ne seront pas repris ici (se reporter aussi aux revues [4-6]).

\section{Combien de gènes impliqués dans la spermatogenèse ?}

Le grand nombre de souris "knock-out" (KO) montrant une atteinte de la spermatogenèse confirme les données de la toxicologie quant à la grande sensibilité testiculaire aux atteintes génétiques. Ainsi, on attendait des perturbations rénales et de la pression sanguine après inactivation du cotransporteur $\mathrm{Na}+\mathrm{K}+-2 \mathrm{Cl}$, mais c'est une stérilité mâle qui a été observée [16]. La mise en évidence de la participation de ces gènes dans la spermatogenèse a ainsi été souvent fortuite. Par exemple, il est apparu que la caséine kinase $2 \alpha^{\prime}$ joue un rôle important dans la morphogenèse des têtes des spermatides, alors qu'il était attendu des anomalies neurologiques [20]. Cela montre que de très nombreux facteurs restent à identifier, $\mathrm{y}$ compris des isoformes de divers facteurs spécifiques des gamètes mâles.

A l'inverse, en ciblant un gène présumé important pour la spermatogenèse, on a parfois eu la surprise de conserver une fertilité masculine. On découvre ainsi que des histones peuvent 
compenser la fonction de l'histone 1 spécifique du testicule (H1t), puisque son inactivation ne perturbe pas la spermatogenèse [11].

\section{La prophase méiotique : une étape parti- culièrement sensible aux modifications métaboliques et au choc thermique}

Les spermatocytes I en prophase méiotique sont particulièrement sensibles à des modifications de l'expression de leurs gènes, notamment ceux impliqués dans la recombinaison. Dans ce cas, ils sont éliminés, ce qui conduit à une déplétion des tubes séminifères. Ces modèles ont donc permis de confirmer l'existence de mécanismes de contrôle, notamment au stade pachytène, afin d'empêcher la transmission à la descendance de cellules germinales génétiquement altérées. L'activation de ces systèmes de contrôle commande le déclenchement d'une mort cellulaire programmée. Par la suite, les cellules de Sertoli se détachent ou se vacuolisent, confirmant l'importance des inter-dépendances entre les cellules somatiques et germinales.

Un autre apport de cette technique concerne donc aussi l'importance, pour les cellules germinales, des gènes de l'évitement de transformations malignes (supresseurs de tumeurs) comme p53 ou des gènes impliqués dans les processus d'induction de l'apoptose comme Bax, et Apaf-1 ou de protection contre l'apoptose, comme ceux de la famille bcl2 (revue dans $[4,6])$.

Le spermatocyte I apparaît aussi être la cellule cible de la réponse au choc thermique. Ainsi l'expression d'une forme activée du facteur de transcription de réponse au stress thermique (HSF1) entraîne-t-elle la mort par apoptose des pachytènes tardifs [13].

\section{Mise en évidence de différences entre méioses mâle et femelle pour des événe- ments apparemment similaires}

Les souris KO permettent de répertorier les gènes impliqués dans la recombinaison et de contrôler la fonction de ces gènes pour l'appariement des chromosomes. Elles révèlent qu'il existe bien des facteurs communs aux deux sexes, comme on pouvait s'y attendre. Mais elles montrent aussi qu'il existe des facteurs spécifiques d'un sexe pour des événements apparemment similaires. Nous prendrons deux exemples particulièrement inattendus. Linactivation du facteur $P m s 2$ de réparation du DNA après recombinaison, n'entraîne un arrêt méiotique que chez le mâle [1], contrairement à l'inactivation du facteur de réparation Mlh1 qui provoque un arrêt méiotique dans les deux sexes [3]. Linactivation de $S c p 3$ codant pour un composant structural majeur des complexes synaptonémaux, ne perturbe que la méioses mâle [22].

\section{L'analyse fonctionnelle des facteurs de condensation du noyau du spermatozoïde}

Bien d'autres aspects de la différenciation des cellules germinales peuvent être abordés par génie génétique, comme par exemple le processus de remplacement des histones par les protéines de transition puis par les protamines au cours de la spermiogenèse (revue dans [6]). L'inactivation de $H R 6 B$ codant pour la dégradation des histones conduit à d'importantes anomalies de la forme du noyau et à une stérilité [17]. Des perturbations dans la régulation de l'expression des gènes des protéines de transition (TPs) ou des protamines conduisent à un arrêt de la spermiogenèse. Par contre, la suppression de l'expression de TP1 n'empêche pas la condensation de la chromatine et seules quelques têtes spermatiques ont une forme anormale. De façon encore inexpliquée, la mobilité est très affectée et seulement $40 \%$ des males sont fertiles avec d'ailleurs des performances abaissées [21]. Enfin, l'inactivation de TP2 n'a, apparemment, pas d'incidence sur la fertilité chez la souris [21].

\section{Découverte de gènes impliqués dans la morphogenèse du flagelle $\mathrm{du}$ spermatozoïde}

Les souris KO sont aussi des modèles pour détecter des facteurs impliqués dans l'organisation et la réorganisation du cytosquelette pendant la spermiogenèse. Nous ne citerons que deux exemples. Linactivation d'un gène pour un composant des jonctions d'ancrage intercellulaire, Nectin-2, a provoqué des anomalies majeures des spermatozoïdes, notamment au niveau flagellaire. Ces anomalies pourraient s'expliquer par la possibilité que 
cette nectine contribue à déplacer l'actine pour la formation du flagelle cours de sa morphogenèse normale [2]. Des anomalies de l'assemblage et de l'allongement des composants flagellaires sont retrouvés après inactivation de $J u n D$, un gène dont la fonction reste encore à élucider [18].

\section{Surprises au niveau de la notion de barrière hémato-testiculaire}

Linactivation d'un gène codant pour un composant majeur des jonctions serrées, Osp / claudin-11, a tout naturellement entraîné le détachement des cellules de Sertoli dans la lumière des tubes séminifères. Pourtant, aucun processus d'autoimmunité ne s'est produit et aucune cellule d'origine sanguine ni aucun signe d'inflammation n'a été observé chez les souris concernées [7]. De plus, l'expression anormale de l'interleukine 2 humaine chez la souris conduit à l'infiltration de lymphocytes dans les tissus cérébraux et la peau, mais pas dans les testicules, qui pourtant développent une déplétion germinale [15].

\section{Des retombées pour la biologie de la reproduction en général}

Une vingtaine de gènes peuvent être à présent répertoriés, dont l'inactivation n'a pas seulement conduit à une perturbation de la spermatogenèse, mais aussi à des anomalies à d'autres niveaux des organes de la reproduction tant masculine (conduits, glandes annexes) que féminine (glande mammaire, utérus, placenta), ou au niveau du produit de la fécondation (implantation, survie embryonnaire), ou même du comportement sexuel et maternel (revue dans [6]).

\section{Gènes de la méiose et gènes de susceptibilité au cancer}

Les souris KO permettent d'identifier les gènes qui sont impliqués à la fois dans le maintien de l'intégrité du génome des cellules somatiques et dans les recombinaisons génétiques. On peut ainsi constater qu'il existe une variété de situations avec des gènes spécifiques de tissus, des gènes communs aux cellules lymphoïdes et germinales et aussi, dans ce deuxième cas, des spécificités de sexe. Une retombée majeure est l'obtention de modèles animaux pour des syn- dromes humains, comme pour le gène Atm de l'Ataxie télangiectasie [19]. Par transgenèse, on peut aussi découvrir des gènes qui pourraient avoir un rôle dans l'apparition de tumeurs testiculaires. Cela a été le cas en surexprimant $G D N F$, un facteur neurotrophique exprimé dans les spermatogonies [12].

\section{Maladies associées}

Des atteintes somatiques sont aussi apparues pour une vingtaine de gènes inactivés par génie génétique, et qui ont entraînés une perturbation de la spermatogenèse. Elles ne se limitent pas à des immunodéficiences mais peuvent atteindre des tissus très variés (revue dans [6]). Cela ne saurait nous surprendre dans la mesure où un certain nombre de souris de laboratoire à mutation spontanée présentent des maladies associées à une stérilité masculine.

\section{Différences fonctionnelles inter- spécifiques de gènes homologues}

Bien entendu, comme tout modèle animal, quelques réserves peuvent être faites. Nous prendrons comme seul exemple la question d'une homologie de séquence d'un gène d'une espèce à l'autre, qui ne signifie pas toujours une fonction similaire pour les raisons suivantes : 1) du fait d'une homologie restreinte à un seul domaine (c'est le cas des membres de super-familles) ; 2) la fonction du gène peut être conditionnée par le stade d'expression et/ou la localisation du produit et 3) le produit d'un gène peut avoir pour fonction de réguler la translation de différent facteurs, comme les protéines associées aux mRNAs (exemple les gènes $\mathrm{DAZ}$ ) [8]. Ces divergences fonctionnelles entre gènes homologues trouvent un exemple qui pourrait être un modèle : l'inactivation de Insl3 provoque une cryptorchidie chez la souris, mais aucune anomalie significative de ce gène n'est retrouvée après l'étude d'hommes présentant cette affection [9].

\section{Le spermatozoïde : une cellule caractéri- sée par une dissociation entre phénotype et génotype}

Les résultats obtenus par inactivation d'un gène sont conditionnés par l'existence de ponts entre les cellules germinales qui permettent le 
passage de mRNAs et de protéines. Seules les souris homozygotes étaient stériles. Donc à l'état hétérozygote, la moitié des spermatozoïdes pressentant un phénotype normal portait un défaut génétique (revue dans [4]).

\section{CONCLUSION}

Bien que les enseignements acquis par génie génétique aient leurs limites, comme toute manipulation expérimentale, il est indéniable que la connaissance de la spermatogenèse des mammifères a fait un bond en avant par cette approche et cette avancée ne fait que commencer. De plus, l'émergence conjointe de cette technique et de la fécondation par microinjection dans l'ovocyte a donné un regain d'intérêt à l'étude de la spermatogenèse.

Remerciements : Le Professeur J.P. DADOUNE (Hôpital Tenon et Université Paris VI, France) est remercié d'avoir été l'inspirateur de la suite donnée aux précédentes revues sur ce thème. De très nombreux auteurs ne peuvent être cités dans cette synthèse limitée en volume. On en trouvera les références dans les revues citées en introduction.

\section{REFERENCES}

1. BAKER S.M., BRONNER C.E., ZHANG L. et al. : Male defective in the DNA mismatch repair gene PMS2 exhibit abnormal chromosome synapsis in meiosis. Cell, 1995, 82 : 309-319.

2. BOUCHARD M.J., DONG Y., McDERMOTT Jr B.M. et al. : Defects in nuclear and cytoskeletal morphology and mitochondrial localization in spermatozoa of mice lacking nectin-2, a component of cell-cell adherens junctions. Mol. Cell. Biol., 2000, 20 : 2865-2873.

3. EDELMANN W., COHEN P.E., KANE M. et al. : Meiotic pachytene arrest in Mlh1-deficient mice. Cell, 1996, 85 : 1125-1134.

4. ESCALIER D. : Mammalian spermatogenesis investigated by genetic engineering. Histol. Histopathol., 1999, $14: 945-958$.

5. ESCALIER D. : What are the germ cell phenotypes from infertile men telling us about spermatogenesis ? Histol. Histopathol., 1999, 14 : 959-971.

6. ESCALIER D. : Impact of genetic engineering on the spermatogenesis understanding (soumis pour publication).

7. GOW A., SOUTHWOOD C.M., Li J.S, et al. : CNS myelin and Sertoli cell tight junction strands are absent in Osp/claudin-11 null mice. Cell, 1999, 99 : 649-659.
8. HOUSTON D.W., KING M.L. : A critical role for Xdazl, a germ plasm-localized RNA, in the differentiation of primordial germ cells in Xenopus. Development, 2000, $127: 447-456$.

9. KRAUSZ C., QUINTANA-MURCI L., FELLOUS M. et $\alpha l$. : Absence of mutations involving the INSL3 gene in human idiopathic cryptorchidism., Mol. Hum. Reprod., 2000, 6 : 298-302.

10. LAMB D.J. : Debate : Is ICSI a genetic time bomb ? Yes. J. Androl., 1999, 20 : 23-32.

11. LIN QC, SIROTKIN A., SKOULTCHI A.I. : Normal spermatogenesis in mice lacking the testis-specific linker histone H1t. Mol. Cell. Biol., 2000, 20 : 21222128.

12. MENG X., LINDAHL M., HYVONEN M.E., et al. : Regulation of cell fate decision of undifferentiated spermatogonia by GDNF. Science, 2000, $287: 1489$ 1493.

13. NAKAI A., SUZUKI M., TANABE M. : Arrest of spermatogenesis in mice expressing an active heat shock transcription factor 1. Embo J., 2000, 19 : 1545-1554.

14. OKABE M., IKAWA M., ASHKENAS J. : Male infertility and the genetics of spermatogenesis. Am. J. Hum. Genet., 1998, 62 : 1274-1281.

15. OHTA M., MITOMI T., KIMURA M. et al : Anomalies in transgenic mice carrying the human interleukin-2 gene. Tokai J. Exp. Clin. Med., 1990, 15 : 307-315.

16. PACE A.J., LEE E., ATHIRAKUL K. et al. : Failure of spermatogenesis in mouse lines deficient in the $\mathrm{Na}$ +$\mathrm{K}+-2 \mathrm{Cl}$ - cotransporter. J. Clin. Invest., 2000, 105 : 441-450.

17. ROEST H.P., VAN KLAVEREN J., DE WITJ. et al. : Inactivation of the HR6B ubiquitin-conjugating DNA repair enzyme in mice causes male sterility associated with chromatin modification. Cell, 1996, 86, 86 : 799-810.

18. THEPOT D., WEITZMAN J.B., BARRA J. et al. : Targeted disruption of the murine jun $\mathrm{D}$ gene results in multiple defects in male reproduction function. Development, 2000,127:143-153.

19. XU Y., ASHLEY T., BRAINERD E.E. et al. : Targeted disruption of ATM leads to growth retardation, chromosomal fragmentation during meiosis, immune defects and thymic lymphoma. Genes Dev., 1996, 10 : 2411-2422.

20. XU X., TOSELLI P.A., RUSSELL L.D. et al. : Globozoospermia in mice lacking the casein kinase II $\alpha$ ' catalytic subunit. Nat. Genet., 1999, 23 : 118-121.

21. YU Y.E., ZHANG Y., UNNI E. et al. : Abnormal spermatogenesis and reduced fertility in transition nuclear protein 1- deficient mice. Proc. Natl. Acad. Sci. USA. 2000, 97 : 4683-4688.

22. YUAN L., LIU J.G., ZHAO J. et al. : The murine SCP3 gene is required for synaptonemal complex assembly, chromosome synapsis and male fertility. Mol. Cell., $2000,5: 73-83$. 


\section{ABSTRACT \\ GENETIC ENGINEERING AND \\ SPERMATOGENESIS}

\section{ESCALIER}

The knowledge of mammal spermatogenesis takes great advantage of the powerful method of functional analysis by genetic engineering. This method allows to study the factors implicated in meiosis depending on the sex and their possible relationships with tumorigenesis and apoptosis. It can be distinguished the factors possibly involved in sterilities and those that can be compensated by genetic redundancy. The mechanisms of spermiogenesis can be dissected, as can be those of the heat shock response and the hemato-testicular barrier. Data extent to the biology of reproduction at various levels in both sexes and to the question of sterility-associated diseases. Knock-out mice also show the importance of the male germ cell genotype/phenotype dissociation in hemizygous. As an experimental approach, the method knows some limitations such as the differences between species in the function and expression of homologous genes. The unexpected failures of spermatogenesis found in many cases supports the notion that spermatogenesis is very sensitive to genetic damages and show that many genes have to be investigated.

Key words : knockout mice, meiosis, spermatogenesis, spermatozoa, testis. 